\title{
The study of the effect of concentration of the agar-agar solution on the Rheological and thermo rheological behaviour
}

\author{
Naima.Sahraoui ${ }^{1}$, Hacina Abchiche ${ }^{2,}$ Mounir Mellal $^{3}$, Sadjia Bertouche ${ }^{4}$, Lyna Tebachi $^{5}$, \\ Amel Mameri ${ }^{6}$ \\ Laboratory of Transfer Phenomena, Faculty of Mechanical and Process Engineering, University of Science and \\ Technology Houari Boumediene, El Alia, BP32, Bab Ezzouar, 16111, Algiers, Algeria
}

\begin{abstract}
:
The purpose of this work is to study the parameters that influe the rheological behavior of a complex fluid (agar agar solution), on a capillary remoter., the rheological properties of agar solution were studied, in particular the concentration's effect $(0,1 \%, 0,15 \%, 0,17 \%, 0,2 \%)$, and temperature $\left(20^{\circ} \mathrm{C}, 30^{\circ} \mathrm{C}, 40^{\circ} \mathrm{C}, 50^{\circ} \mathrm{C}, 60^{\circ} \mathrm{C}, 70^{\circ} \mathrm{C}\right)$ on the rheological behavior of the agar solution. A comparative study of various rheological laws (Herschel-Buckley, Cross, Oswald De Waele, Bingham) was made, to establish a model that presents the rheological behavior which applies to different samples of agar solution, based on the coefficient of correlation. Results indicated that the rheological behavior of agar solution depends on the range of shear rate used: for low shear rate (lower than $6.2 \mathrm{~s}^{-1}$ ) the solution of agar can be modeled by an Ostwald-De Waele-type power-law, but for the high shear rate (higher than 6.2 $\mathrm{s}^{-1}$ ) the solution of agar can be described by Bingham's model. The concentration's and temperature's effect of agar solution on the two models studies' parameters (Ostwald-De Waele, Bingham) was studied.
\end{abstract}

Keywords: Agar- agar, Bingham, Ostwald-De-Waele Rheological behavior, Thermo rheological behavior

\section{Introduction}

Today, many pharmaceutical, food and cosmetic industries use natural raw materials extracted from plants or animals. there is a real need to go back to the natural world.: whether it is to recover health or to better preservation. It is obvious that a natural product is necessarily better than a conventional one, it is almost a question of common sense. Safer, better quality (for example: natural cosmetics usually contain more active ingredients than conventional ones), without additives, dyes and synthetic fragrances. Agar-agar is a food gallant extracted from red algae, very rich in minerals, including calcium and iron. The gelling effect is much stronger than that of animal gelatin ( 7 to 8 times) and of course healthier. It is ideal for 


\title{
2nd International Conference on Research in Applied Science
}

\author{
30 October - 01 November 2019 \\ Milan, Italy
}

slimming treatments with the advantage of not changing the tastes and colours. It is calming, and it heals the intestinal wall damaged by acidic substances or drugs and has both laxative and anti-diarrheal action. Also Anti oxidant, moisturizer, participating in cell renewal. It stabilizes the texture of pharmaceutical and cosmetic creams. The determination of the rheological properties of agar-agar solutions is essential to adapt the use of this type of solutions to industrial conditions.

This work is part of a project whose objective is to study the parameters that influence the rheological behaviour of agar-agar solutions; including the effect of concentration and temperature.

\section{Materials and methods}

\subsection{Product used (agar solution)Submitting}

The different physicochemical characteristics of agar- agar are grouped in the table .1 .

Table 1: Physicochemical characteristics of agar-agar.

\begin{tabular}{|c|c|}
\hline Function & Texturing gelling agent \\
\hline Aspect & powder \\
\hline Composition & AGAR-AGAR pure at $100 \%$ \\
\hline Alcoholic degree & $0 \%$ \\
\hline Solubility & Dispersible in cold, good solubility at $90^{\circ} \mathrm{C}$ \\
\hline Dosage & Between 0.2 et $1.5 \%$ Maximum \\
\hline pH & 6 to 8 in a $1 \%$ aqueous solution \\
\hline
\end{tabular}

\subsection{Protocol for the preparation of agar-agar solutions}

For this study, we have prepared 4 solutions of different concentration $(0.1-0.15-0.17$ and $0.2 \%)$. The experimental protocol adopted for the preparation of 501 agar-agar solution is detailed below. The one at a concentration of $0.1 \%$ (the same protocol is followed for the other concentrations):

$\checkmark$ Prepare 50 liters of distilled water in a tank.

$\checkmark$ Weigh $50 \mathrm{~g}$ of agar-agar powder.

$\checkmark$ Heat the distilled water to $90^{\circ} \mathrm{C}$.

$\checkmark$ Add radually the powder into distilled water while stirring (using a helical stirrer) until the material dissolves completely.

$\checkmark$ Allow the mixture to sit in a tank at room temperature for a minimum of 6 hours to remove any air bubbles in the solution. 


\title{
2nd International Conference on Research in Applied Science
}

\author{
30 October - 01 November 2019 \\ Milan, Italy
}

\subsection{Rheological characterization}

To characterize the rheological properties of non-Newtonian fluids (agar-agar solution, we adopted a method based on the measurement of pressure drop and flow rate. The experimental set-up conceived in our laboratory, which served as support for the present study, is illustrated in Fig.1.

Fig. 1: Schematic

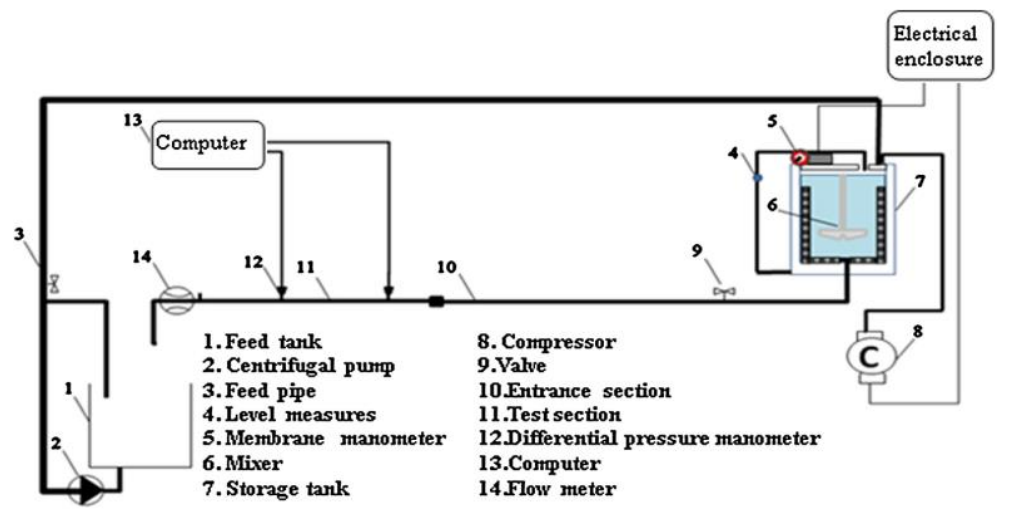

the experimental setup [1]

diagram of

It consists essentially of: The storage bins up-and downstream, the fluid is thus aspirated from the upstream tank by a downstream pump unit equipped with a speed regulator toward the upstream tank with overpressure with respect to atmospheric pressure, a compressor, with circular horizontal pipes of $10 \mathrm{~mm}$ diameters placed at the end of a 3-m-long cylindrical pipe, used to establish the flow regime. The test lines are equipped with pressure sensors for measuring pressure. Flow measurement is carried out by means of an electromagnetic flowmeter.

In order to study the effect of temperature on the rheological behaviour of the non-Newtonian fluid, we designed heating pipes to heat the fluid, Fig. 2. Heating is carried out by a coaxial heating wire (Thermocoax) wound on the measuring lines. It consists of a Nickel-Chromium conductor wire placed in a stainless steel sheath. The wire is insulated from the sheath with Teflon tape (75\% virgin PTFE, $25 \%$ fibreglass). All pipes are effectively insulated with a glass wool jacket. The heating pipe shown in Figure II is equipped with 6 temperature connections, spaced $0.5 \mathrm{~m}$ apart and 3 pressure connections. Fig. 2: General diagram of the heating pipe

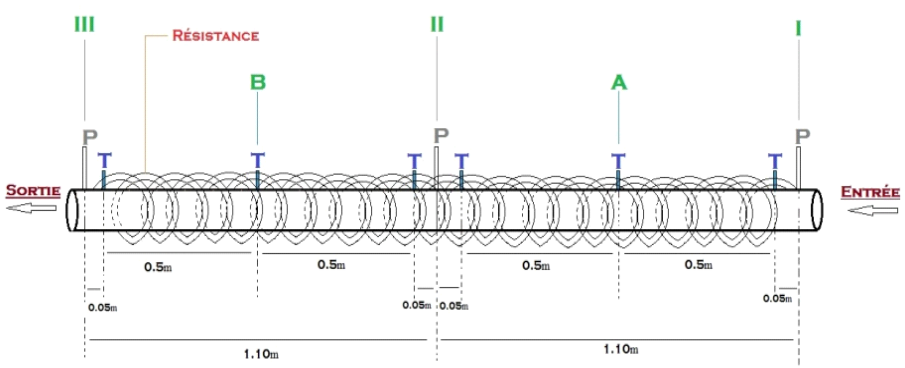




\section{2nd International Conference on Research in Applied Science}

30 October - 01 November 2019

Milan, Italy

\subsection{Modelling the rheological behaviour of flowing fluids}

In a horizontal circular pipe of diameter $\mathrm{d}$ and length $\mathrm{L}$, the pressure loss is related to the shear stress at the wall $\mathrm{u}_{\mathrm{p}}$ by the following relationship:

$$
\tau_{w}=\frac{\Delta p R}{2 L}
$$

The flow rate is related to the shear rate using the relationship of Rabinowitsch-Mooney [2]. It should be noted that the Mooney-Rabinowitsch relationship is valid for all time-independent fluids in laminar flow and assumes that there is no wall effect, due to the zero adhesion speed.

The flow rate is related to the shear rate using the Rabinowitsch-Mooney relationship [2]. It should be noted that the Mooney-Rabino

witch relationship is valid for all time-independent fluids in laminar flow and assumes that there is no wall effect, due to the zero adhesion speed

The wall shear stress ( $\tau \mathrm{w})$ and the apparent wall shear rate $\left(\gamma_{\mathrm{app}}\right)$ were obtained from equations (1) and (2):

$$
\dot{\gamma}=\frac{4 Q^{\prime}}{\pi D^{3}}\left[\frac{3 m+1}{4 m}\right]
$$

With:

$$
m=\frac{\operatorname{dlog}\left(\frac{\Delta p}{L}\right)}{\operatorname{dlog}(Q)}
$$

From these relationships we can determine for each flow the shear rate, just draw $\log (\Delta \mathrm{p} / \mathrm{L})$ as a function of $\log (\mathrm{Q})$, the slope gives the value of $(\mathrm{m})$, this value is then injected into equation (2).

\section{Results and discussion}

\subsection{Choice of rheological model}

In order to choose the rheological model representing the agar-agar solution in the range of shear rates studied, we carry out a comparative study between the three rheological laws to follow: Herschel-Bulckley, Bingham, Ostwald-De-Waele. To choose the correct mathematical model, it was based on the highest correlation coefficient $\mathrm{R}^{2}$. The results obtained for a solution of agar-agar at $0.1 \%$ at $20^{\circ} \mathrm{C}$ are show in Fig. 3 . 


\section{2nd International Conference on Research in Applied Science}

\section{October - 01 November 2019}

Milan, Italy

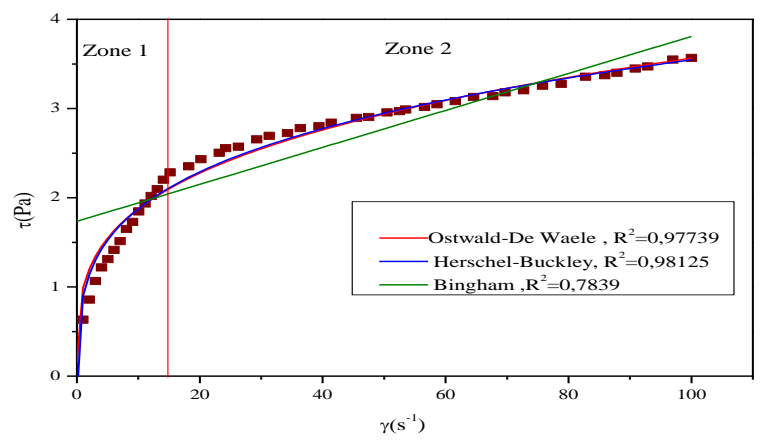

Fig.3: Comparison between the experimental points and those given by three Rheological models. $\left(\mathrm{C}=0.1 \%, \mathrm{~T}=20^{\circ} \mathrm{C}\right)$.

The values of the correlation coefficient $\mathrm{R}^{2}$ for the four concentrations of the agar solutions studied are shown in Table 2.

Table 2: Correlation coefficient for the three rheological laws.

\begin{tabular}{ccccc}
\hline$C(\%$, & $\begin{array}{c}R^{2} \\
\text { Bingham }\end{array}$ & $\begin{array}{c}R^{2} \text { Ostwald }- \\
\text { De Waele }\end{array}$ & $\begin{array}{c}R^{2} \text { Herschel }- \\
\text { Buckley }\end{array}$ & $\tau_{c}$ \\
0,10 & 0,977 & 0,783 & 0,981 & $\mathbf{- 0 , 5 1 5}$ \\
0,15 & 0,569 & 0,897 & 0,888 & $\mathbf{- 1 , 6 6 1}$ \\
0,17 & 0,981 & 0,995 & 0,989 & $\mathbf{- 0 , 2 6 2}$ \\
0,20 & 0,498 & 0,880 & 0,867 & $\mathbf{- 5 , 4 2 1}$ \\
\hline
\end{tabular}

We note that from the previous table, the correlation coefficient $\mathrm{R}^{2}$ is higher for the Herschel-Bulkley model. Nevertheless, this one is discarded; because of the threshold stress obtained, which is negative, it is physically unacceptable. We have to choose between the Bingham model and the Ostwald-De Waele model. The Bingham model does not represent well the experimental points in the whole studied shear range $\left(\mathrm{R}^{2}=0.784\right)$ and that of Ostwald - De Waele does not seem very adequate also beyond a certain shear range. Seeing the fig3, the presence of two zones can be distinguished: a region with a very low shear rate $\left(\gamma<0.6 \mathrm{~s}^{-1}\right)$, the fluid has a shear-thinning behavior, a second region $(\gamma>0.6$ $\left.\mathrm{s}^{-1}\right)$, the fluid has a plastic behavior.

\subsection{Study of the parameters influencing the rheological behavior of agar -agar solution in the first shear zone}

In the following, we study the effects of the concentration and temperature of agar-agar on the rheological behaviour of prepared solutions. 


\title{
2nd International Conference on Research in Applied Science
}

\author{
30 October - 01 November 2019 \\ Milan, Italy
}

\subsection{Effect of agar-agar concentration}

The following figure groups the rheograms obtained for four concentrations of agar-agar in the shearing range from 0 to $6.2 \mathrm{~s}^{-1}$.

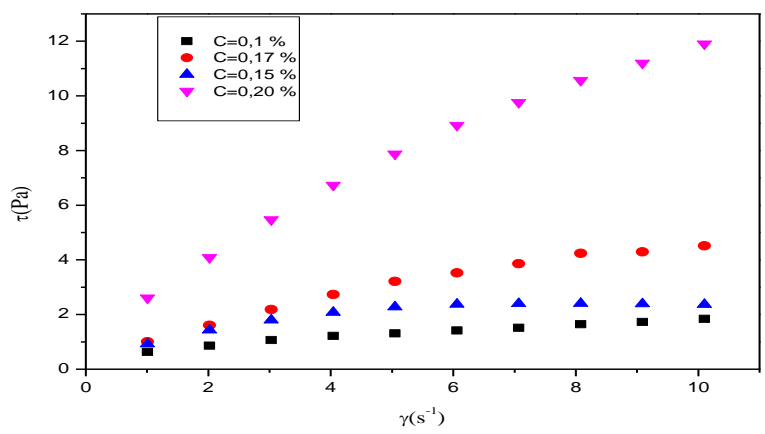

Fig.4: Variation of the shear stress as a function of shear rate, $(\gamma<0,6 \mathrm{~s}-1), \mathrm{T}=20^{\circ} \mathrm{C}$.

From the rheograms obtained in the previous figure, we find that at a given shear rate, the shear stress increases with increasing concentration. Modelling the behaviour of agar-agar solutions by Ostwald-De Waele type law (given by the following equation $\tau=k \dot{\gamma}^{n}$ ), enables us to determine the indices of consistency" $k$ " and the flow index "n". Table 3 groups the results according to the concentrations of agar-agar.

Table3: Rheological laws of Agar-agar solutions at different concentrations. $\left(\dot{\gamma}<0,6 s^{-1}\right)$

\begin{tabular}{ccc}
\hline$\%$ & $n$ & $k\left(P a \cdot s^{n}\right)$ \\
0,10 & 0,648 & 0,627 \\
0,15 & 0,558 & 1,126 \\
0.17 & 0,460 & 1.190 \\
0,20 & 0.339 & 2,709 \\
\hline
\end{tabular}

The previous results show that the flow index behaves differently with the fluid concentration. We note on the one hand that the flow index is less than 1; thus indicating the pseudoplastic character of the agar-agar solution and on the other hand, we see a decreasing relationship between flow index and concentration it may be due to the strengthening of macromolecular; when the concentration increases which makes the fluid away from the newtonian behavior hence the reduction of flow index.

As for the dependence of the consistency index on concentration, we observe an increases of ' $\mathrm{k}$ ' as a function of concentration; this result is due to the entanglement of polymer chains. 


\title{
2nd International Conference on Research in Applied Science
}

\author{
30 October - 01 November 2019 \\ Milan, Italy
}

\subsection{Effect of temperature}

In order to study the influence of the temperature of aqueous agar-agar solutions on their rheological behaviour, we will monitor the evolution of the shear stress of these solutions as a function of the shear rate at different temperatures $\left(20.30,40,50,60\right.$ and $\left.70^{\circ} \mathrm{C}\right)$. The results are shown in Fig. 5.

Fig.5: Variation of the as a function of shear

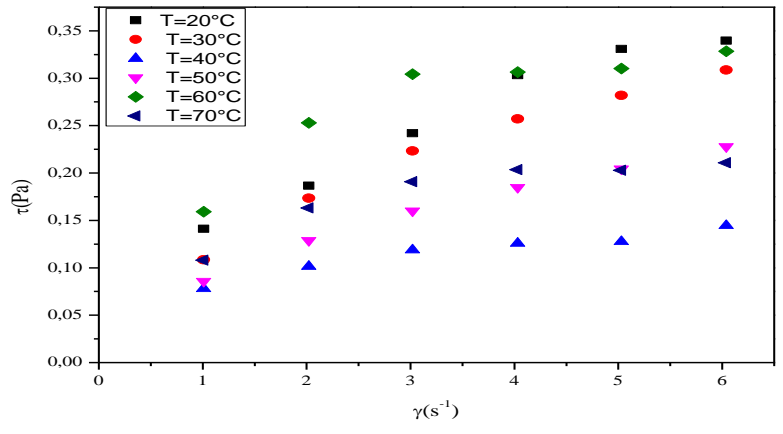

$\left.s^{-1}\right), C=0,17 \%$. shear stress rate, $(\dot{\gamma}<6.2$

The previous figure allows us to determine the consistency ' $\mathrm{k}$ ' and behaviour ' $\mathrm{n}$ ' indices of the agar-agar solution at different temperatures. Table 4 groups the results.

Table 4: Evolution of consistency" $\mathrm{k}$ " and the flow index " $\mathrm{n}$ " at different temperatures, $\mathrm{C}=0,17 \%$

\begin{tabular}{ccccccc}
\hline$T\left({ }^{\circ} \mathrm{C}\right)$ & 20 & 30 & 40 & 50 & 60 & 70 \\
$n(-)$ & 0,454 & 0,480 & 0,51 & 0,457 & 0,37 & 0,175 \\
$\boldsymbol{k}\left(\boldsymbol{P a} \cdot \boldsymbol{s}^{\boldsymbol{n}}\right)$ & 0,765 & 0,50 & 0,316 & 0,314 & 0,26 & 0,177
\end{tabular}

The previous results show that the flow index behaves differently with the fluid temperature. We note on the one hand that the flow index is less than 1; thus indicating the pseudoplastic character of the agar-agar solution and on the other hand, the flow index does not react in the same way to the change in temperature. We notice a slight increase in the flow index for temperatures ranging from 20 to $40^{\circ} \mathrm{C}$, which may be due to the disentanglement of polymer chains. For temperatures ranging from 40 to $70^{\circ} \mathrm{C}$, we see a decreasing relationship between flow index and temperature, which may be due to the strengthening of macromolecular chains in agar- agar solutions above $40^{\circ} \mathrm{C}$.

As for the dependence of the consistency index on temperature, we observe a decrease of ' $\mathrm{k}$ ' as a function of temperature, this decrease is more accentuated for temperatures ranging from 20 to $40{ }^{\circ} \mathrm{C}$; this result is due to the disentanglement of polymer chains, thus explaining the increase in the flow index as well. On the other hand, above $40^{\circ} \mathrm{C}$ the decrease in the consistency index with temperature is less sensitive, this can be explained this time by the entanglement of polymeric chains with the increase in temperature; which means that above $40^{\circ} \mathrm{C}$ the flow index has decreased. 


\section{2nd International Conference on Research in Applied Science}

30 October - 01 November 2019

Milan, Italy

3.3. Study of the parameters influencing the rheological behaviour of agar -agar solution in the second shear zone

\subsection{Effect of agar-agar concentration}

The figure 6 represents the evolution of the shear stress versus shear rate of the second study zone ( 6.2 to $\left.100 \mathrm{~s}^{-1}\right)$ at different concentrations of the agar agar solution.

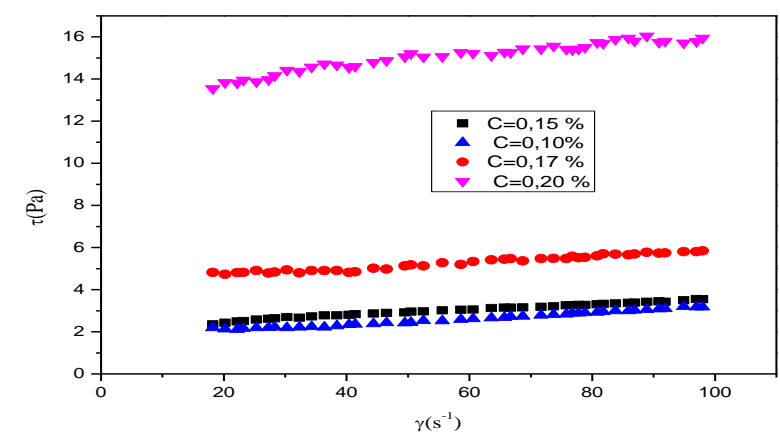

Fig.6 : Variation of the shear stress as a function of shear rate, $\left(\dot{\gamma}>0,6 \mathrm{~s}^{-1}\right), T=20^{\circ} \mathrm{C}$.

From the rheograms obtained in the previous figure, we can see that at a given shear rate, the shear stress increases with increasing concentration. Modelling the behaviour of agar-agar solutions by a Bingham law (of the form $\tau=\tau_{c}+\eta_{p} \dot{\gamma}$ ) allows us to determine critical stress and plastic viscosity $\eta_{\mathrm{p}}$. Table 5 groups the results according to the mass concentrations of agar-agar.

Table5: Evolution of the critical constraint $\tau_{c}$ and plastic viscosity $\eta_{p}$ at different concentrations, $T=20^{\circ} \mathrm{C}$.

\begin{tabular}{ccc}
\hline$C \%$ & $\boldsymbol{\eta}_{\boldsymbol{p}}(\boldsymbol{P a} . \boldsymbol{s})$ & $\boldsymbol{\tau}_{\boldsymbol{c}}(\mathrm{Pa})$ \\
0.10 & 0.013 & 1.786 \\
0.15 & 0.014 & 2.267 \\
0.17 & 0.016 & 4.436 \\
0.20 & 0.027 & 13.455 \\
\hline
\end{tabular}

We note that the increase in the concentration of agar has a positive effect on the critical stress and plastic viscosity, which results in the formation of a polymer network, all the more rigid as the concentration of the gelling agent is high, hence the increase in plastic viscosity. The polymer network requires high pressure to break it, which explains an increase in the critical stress. 


\title{
2nd International Conference on Research in Applied Science
}

\author{
30 October - 01 November 2019 \\ Milan, Italy
}

\subsection{Effect of temperature}

Shows the variation of shear stress as a function of shear rate for different temperatures of agar-agar solutions $\left(20,30,40,50,50,60\right.$ and $\left.70^{\circ} \mathrm{C}\right)$. The previous figure allows us to determine the critical stress and plastic viscosity of agar-agar solutions for each study temperature. Table 6 groups the different results.

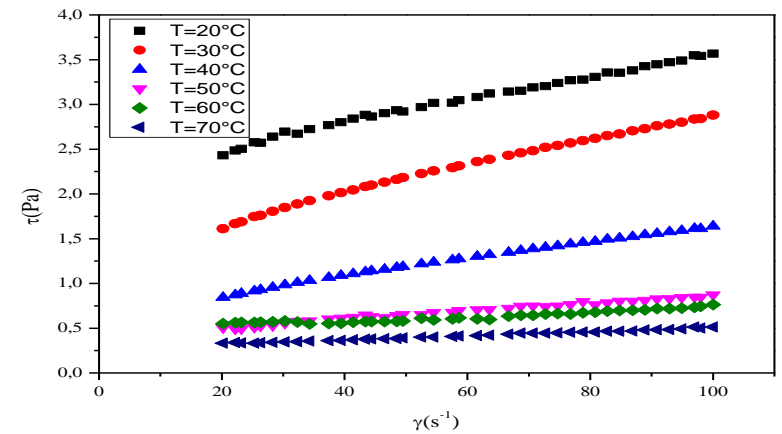

Fig.7: Variation of the shear stress as a function of shear rate at different temperature, $\left(\boldsymbol{\gamma}>0,6 \mathrm{~s}^{-1}\right)$, $C=0,10 \%$.

Table6: Evolution of critical stress and plastic viscosity $\eta_{p}$ at different temperatures. $C=0,10 \%$.

\begin{tabular}{|c|c|c|c|c|c|c|}
\hline $\mathbf{T}\left({ }^{\circ} \mathbf{C}\right)$ & $\mathbf{2 0}$ & $\mathbf{3 0}$ & $\mathbf{4 0}$ & $\mathbf{5 0}$ & $\mathbf{6 0}$ & $\mathbf{7 0}$ \\
\hline $\boldsymbol{\eta}$ (Pa. s) & 0.010 & 0.0075 & 0,006 & 0,0039 & 0,0028 & 0,0018 \\
\hline $\boldsymbol{\tau}_{\boldsymbol{c}}(\mathbf{P a})$ & 0.253 & 0.216 & 0,189 & 0,145 & 0,104 & 0,06 \\
\hline
\end{tabular}

We observe that the critical constraint $\tau_{c}$ and plastic viscosity $\eta_{\mathrm{p}}$ at high shear rates $(\boldsymbol{\gamma}>0,6$ $\mathrm{s}^{-1}$ ), decrease with increasing temperature. This is probably due to the coupled effect of the shearing of the polymer chains and the increase in temperature; which makes the chains become less rigid, hence the fluid tends to flow more easily, hence the critical stress reduced and also the plastic viscosity.

\section{Conclusions}

The experimental facility designed at the Transfer Phenomena Laboratory was used to characterize the rheological behavior of agar-agar solutions. Measurements of pressure and volume flow gradients allowed us to trace the rheological law of the fluid using the Rabinowitsch - Mooney treatment.

Results indicated that the rheological behavior of agar solution depends on the range of shear rate used: for low shear rate (lower than $6.2 \mathrm{~s}^{-1}$ ) the solution of agar agar can be modeled by an Ostwald-De Waele-type power-law. The flow index is less than 1; thus indicating the pseudoplastic character of the agar-agar solution and we see a decreasing relationship between flow index and concentration, the consistency index increases a function of 


\section{2nd International Conference on Research in Applied Science}

\section{October - 01 November 2019}

Milan, Italy

concentration. The flow index does not react in the same way to the change in temperature. We notice a slight increase in the flow index for temperatures ranging from 20 to $40^{\circ} \mathrm{C}$ but for temperatures ranging from 40 to $70^{\circ} \mathrm{C}$, we see a decreasing relationship between flow index and temperature.

We observe a decrease of ' $\mathrm{k}$ ' as a function of temperature, this decrease is more accentuated for temperatures ranging from 20 to $40{ }^{\circ} \mathrm{C}$. On the other hand, above $40^{\circ} \mathrm{C}$ the decrease in the consistency index with temperature is less sensitive.

For the high shear rate (higher than $6.2 \mathrm{~s}^{-1}$ ) the solution of agar agar can be described by Bingham's model,

the increase in the concentration of agar agar has a positive effect on the critical stress and plastic viscosity and the critical constraint $\tau_{c}$ and plastic viscosity $\eta_{p}$ at high shear rates $(\boldsymbol{\gamma}>$ $\left.0,6 \mathrm{~s}^{-1}\right)$,decrease with increasing temperature.

\section{Acknowledgements}

The authors wish to thank Mr Belamiri Samir for his invaluable assistance to the realization of the experimental installation.

\section{References}

[1] H. Abchiche, M. Mellal, A. Bensakhria and M. Trari, "Comparative study of correction methods of wall slip effects for CMC solutions," C. R. Mecanique vol 343 pp.322-330 , 2015

[2] MOONEY, M.«Explicit formulas for slip and fluidity », J. Rheol., 2, 210-222 (1931)

[3] C. Y. Lin, M. Wu, J. A. Bloom, I. J. Cox, and M. Miller, "Rotation, scale, and translation resilient public watermarking for images," IEEE Trans. Image Process., vol. 10, no. 5, pp. 767-782, May 2001. 